\title{
A study of bladder dysfunction in diabetes mellitus
}

\begin{abstract}
Diabetic cystopathy was initially described as a complication of diabetes, characterized by an increase in bladder capacity and in post voiding residual volumes, accompanied by a decreased in bladder contraction and vesical sensation. Today, the term includes symptoms of overactive bladder.

The main of this study is to evaluate the prevalence of urinary symptoms in the diabetic population, to compare it with the general population and to understand the impact that these symptoms have on this population.

Methods: An inquiry of urinary symptoms was done to the diabetic patients followed in our hospital, being excluded patients with other comorbidities eventually responsible for cystopathy. We also evaluated the impact of symptoms on the daily basis. From a number of 400 patients, 151 were included in our study. A similar number of a control healthy population was inquired, respecting the same protocol. The statistically study was made with nonparametric test.

Results: From the 151 patients evaluated, 76 were female. Our population included 52 DM1 and 99 DM2 patients, with an average of HbA1c: $8.14 \%$ and of $15 y$ years of diabetes duration. 64/151(42\%) had more than one diabetes chronic complication and $35 / 151(23 \%)$ had symptoms of stress urinary incontinence. $114 / 151(75 \%)$ patients had more than one symptom of bladder dysfunction on the storage phase and 53/151(35\%) had symptoms of voiding dysfunction, with only 28 patients with no symptoms of urinary dysfunction. Comparing to our healthy population we can attribute the bladder dysfunction symptoms to diabetes mellitus.

The impact of the urinary symptoms on their daily basis was, on average, seven on a scale of $0-10$

Conclusion: Diabetic cystopathy is a highly prevalent complication. For a correct evaluation other causes must be ruled out; a good clinical history with an inquiry of urinary symptoms help to characterize the disease stage, which is decisive for the choice of therapeutic strategy.
\end{abstract}

Keywords: bladder dysfunction, diabetic cystopathy, diabetes, cystopathy
Volume 2 Issue I - 2018

\section{Úrsula Martins, ' Érico Costa, ${ }^{2}$ Lúcia \\ Guedes, ${ }^{2}$ Dulce Silva, ${ }^{3}$ Joana Gomes ${ }^{4}$ Catarina Aguiar Branco ${ }^{5}$}

'Interno de Formação Específica de Medicina Física e de Reabilitação no Centro Hospitalar Entre o Douro e Vouga, Portugal

${ }^{2}$ Interno de Formação Específica de Medicina Interna no Centro Hospitalar Entre o Douro e Vouga, Portugal

${ }^{3}$ Assistente Hospitalar Graduada de Medicina Interna no Centro Hospitalar Entre o Douro e Vouga, Portugal

${ }^{4}$ Assistente Hospitalar Graduada de Medicina Física e de Reabilitação no Centro Hospitalar Entre o Douro e Vouga, Portugal

${ }^{5}$ Assistente Hospitalar Graduada e Directora de Serviço de Medicina Física e de Reabilitação no Centro Hospitalar Entre o Douro e Vouga, Portugal

Correspondence: Úrsula Martins, Interno de Formação Específica de Medicina Física e de Reabilitação no Centro Hospitalar Entre o Douro e Vouga, Portugal, Email ana.ursula.cm@gmail.com

Received: February II, 20I8 | Published: February 23, 2018
Abbreviations: HDL, high-density; LDL, low-density; ICS, international continence society; IUA, international urogynecological association; BMI, body mass index.

\section{Introduction}

Diabetic bladder dysfunction refers to a group of bladder symptoms occurring in diabetic patients, whose urologic complications have a negative effect on their quality of life. It is estimated that over than $50 \%$ of men and women with diabetes have bladder dysfunction. ${ }^{1}$ There is a multifactorial pathophysiology, including changes in detrusor, urothelium, urethra, peripheral and autonomic nervous system. ${ }^{1-3}$ Hyperglycemia and polyuria are the main causes, presenting a determining and distinct role in the pathogenesis of the disease: polyuria as an early impact, as opposed to the late consequences of chronic hyperglycemia. ${ }^{4-6}$ Today, current theories support that a peripheral nervous system dysautonomiais are the main cause, but further research in the area is still lacking. In an early stage, overactive bladder symptoms associated with frequency, nocturia and incontinence are predominant, while diminished bladder sensation, poor contractility, and increased post-void residual urine most likely represents an end stage bladder failure, being less common. ${ }^{3-7}$ In urodynamics, bladder over activity is a consequence of involuntary detrusor contractions during bladder filling phase, with or without incontinence, while the bladder hypo activity is characterized by an increased bladder emptying time, as well as post voiding residual volumes. ${ }^{4}$ Change in repletion sensation can lead to abnormal increase in bladder capacity, also contributing to voiding dysfunction phase. ${ }^{8-10}$ Treatment choice is based on these different disease stages. Concomitant factors, such as benign prostatic hyperplasia, neurological disorders, and aging can also contribute to the vesico-urethral dysfunction, being difficult to understand the specific contribution of diabetes in that cases. ${ }^{1-12}$

\section{Purpose}

The purpose of our study was to investigate the prevalence of bladder dysfunction symptoms in a diabetic population, its relation to other diabetes complications, duration and type of diabetes, comparing to a healthy population. We also evaluated the disease impact in quality of life. 


\section{Materials and methods}

Between July and October of 2015, we evaluated 400 diabetic patients followed in our hospital in a diabetic medical consultation, being included in our study a total of 151 patients. Patients with other comorbidities eventually responsible for cystopathy were excluded. A similar number of a control healthy population (150) was inquired, respecting the same protocol.

Inclusion criteria: Diabetic patients followed in our hospital aged $>18$ years. Nomenclature of symptoms and signs related with lower urinary tract dysfunction was in accordance with the International Urogynecological Association/International Continence Society (IUA/ICS) Standardization of Terminology Reports. ${ }^{\text {? }}$

Exclusion criteria: Presence of bladder tumors, pelvic organ prolapse, urolithiasis, prostatic hyperplasia, drug abuse, neurogenic lower urinary tract dysfunction (dementia, mental retardation, cerebrovascular pathology, demyelinization...) and iatrogenic causes, according to literature ${ }^{1-14}$

\section{Symptoms and signs evaluated}

An inquiry of urinary symptoms was done, concerning storage and voiding symptoms, both for the diabetic patients group, as well as for the control healthy population. Incontinence status was evaluated, as well as urgency, frequency and nocturia. Stress incontinence was considered "the loss of control of your urine when you laugh, cough, or during physical activities". ${ }^{1-7}$ Urgent incontinence was defined as the "loss of control of your urine when you feel urgent to urinate but cannot reach the bathroom in time" ${ }^{1-7}$ Concerning voiding symptoms, the sensation of incomplete emptying was considered, as well as the presence of a weak stream, abdominal straining and intermittency. ${ }^{7}$ Vesical sensation and the difficulty to start the micturion were also questioned. Each positive answer (meaning the presence of the symptom) was quoted with one point, so patients could obtain a maximum punctuation of four points in the storage phase (four questions were done) and five in the voiding phase. It was considered the presence of a disturb in the storage or in the voiding phases, if in each phase there was a punctuation equal or superior to two points. The same inquiry was done to health control population. Parameters evaluated: age, sex, diabetes type and duration, therapy instituted (insulin, oral anti diabetic or both), chronic diabetic complications (nephropathy, retinopathy, peripheric neuropathy, cardiac disease or arterial disease), metabolic syndrome, weight (kilograms), height (meters), waist circumference (centimeters). Body mass index (BMI) was calculated.
Laboratory data obtained: glycosylated hemoglobin (HbA1C), total, high-density (HDL) and low-density (LDL) lipoprotein cholesterol, triglycerides. Metabolic syndrome was defined by the presence of three or more risk factors: waist circumference $>80 \mathrm{~cm}$, diabetes type 2 , serum triglyceride level $>150 \mathrm{mg} / \mathrm{dl}$, systolic blood pressure $>130 \mathrm{mmHg}$ or diastolic blood pressure $>85 \mathrm{mmHg}^{1-15}$ Rethinopathy diagnosis was done by an ophthalmologist; nephropathy was defined as an increased urinary albumin excretion, of $30 \mathrm{mg} / \mathrm{g} \mathrm{Cr}$ or superior. Cardiac and arterial disease was found out, consulting the clinical process. Peripheral neuropathy was assessed by questioning patients about paresthesias, as well as measuring thesensory threshold (proprioceptive, painful and touch) on the feet. ${ }^{1-17}$ In the control healthy population, we just evaluated age, sex and BMI, as we assumed that they had not any other comorbidities.

\section{Statistical analysis}

Statistical analyses were performed using SPSS with nonparametric tests (Wilcoxon signed-rank test, Kolmogorov test, Squared ranks test) The study protocol was approved by the institutional ethics committee: All participants gave their written informed consent.

\section{Results}

From the 151 diabetic patients evaluated, 76 were female and 75 were male, with the mean patient's age of 54 years old. Our population included 52 DM1 and 99 DM2 patients, with HbA1c average: 8.14\% and a mean of 15 years of diabetes duration. 42/151 (28\%) were treated with insulin and 31/151 (20\%) with oral Antidiabetic therapy. Diabetic patient's characteristics are shown in Table 1. Diabetes duration had a positive relation with bladder dysfunction. Although without statistical significance, we found out more symptoms as the disease duration increases; deficient control of the disease (HbA1C > $8 \%$ ) had a positive relationship with bladder dysfunction symptoms $(p<0.05)$. On the other hand, storage symptoms were significantly associated with age $(p<0.05)$, being more prevalent in patients older than 50 years $(p<0.05)$, with female sex $(p<0.05)$, with body mass index $(\mathrm{p}<0.01)$, being more prevalent if higher to $30 \mathrm{~kg} / \mathrm{m} 2$ and to diabetes type $2(\mathrm{p}<0.01)$. From our diabetic patients, 56/151(37\%) had metabolic syndrome and 64/151(42\%) had more than one diabetes chronic complication. 48/151 (32\%) patients had nephropathy and we found a statistically significant relation between renal complication and storage symptoms $(\mathrm{p}<0,05)$. Chronic complications are shown in Table 2. In our study we excluded patients with cerebrovascular disease history; therefore we did not evaluate the impact and relation of vascular disease.

Table I Characteristics of diabetic population: mean values of each item are presented

\begin{tabular}{|c|c|c|c|c|c|c|}
\hline \multirow{2}{*}{$\begin{array}{l}\text { Number } \\
n=151\end{array}$} & & \multirow{2}{*}{$\begin{array}{l}\text { Age (years) } \\
P<0.05\end{array}$} & \multicolumn{2}{|c|}{ Diabetes duration since diagnosis (years) $P<0.05$} & \multirow{2}{*}{$\begin{array}{l}\text { BMI }(\mathrm{Kg} / \mathrm{m} 2) \\
\mathrm{P}<0.01\end{array}$} & \multirow{2}{*}{$\begin{array}{l}\text { Waist circumference } \\
\mathbf{P}<0.01\end{array}$} \\
\hline & & & & & & \\
\hline DMT1 & DMT2 & & & & & \\
\hline 52 & 99 & 54 & 15 & & 29,30 & 104 \\
\hline \multicolumn{7}{|c|}{2 Chronic complications of DM: number $(n)$ of patients } \\
\hline HDL & LDL & TG & HbA1C (\%) & Diabetes treatment $P$ & 0.05 & \\
\hline $\mathbf{P}<0.05$ & $\mathbf{P}<\mathbf{0 . 0 5}$ & $\mathbf{P}<\mathbf{0 . 0 5}$ & $\mathbf{P}<0.05$ & Medications (n) & Insulin (n) & Both (n) \\
\hline 47 & 93 & 150 & $8,14 \%$ & $31(20 \%)$ & $42(28 \%)$ & $78(52 \%)$ \\
\hline
\end{tabular}


In our diabetic population, 38/76 (50\%) women had two or more symptoms of storage phase dysfunction secondary to diabetes. The overall prevalence of stress urinary incontinence was $35 / 151(23 \%)$ and of urgency incontinence was 34/151 (22\%). 114/151(75\%) patients had more than one symptom in the storage phase and 53/151(35\%) had symptoms of voiding dysfunction, with only 28 patients with no symptoms of urinary dysfunction (Table 3 ) Control healthy population was younger, with a mean age of 35 years old, being 86 female and 64 male. This population had less bladder dysfunction symptoms; $36 / 150$ $(24 \%)$ had one or more symptoms in the storage phase, being more frequent in women. The prevalence of urge incontinence was 11/150,
5/150 had mixed incontinence, and none had exclusive symptoms of stress incontinence. Patients' characteristics are shown in table 4 and 5. Comparing to our healthy population, we found a significant relation between bladder dysfunction symptoms and diabetes mellitus, since storage and voiding symptoms were significantly higher in diabetes population $(p<0,05)$. Diabetes has been identified as an important independent risk factor for urinary incontinence. Bladder dysfunction, being an insidious and progressive disease, often goes unnoted. However, patients with bladder symptoms (diabetic or not), report an average change in their daily basis of seven out of 10 , being 10 the worst scoring classification.

Table 3 Prevalence of bladder dysfunction symptoms in the diabetic population

\begin{tabular}{llllllll}
\hline Number (n) & \multicolumn{2}{l}{ Storage symptoms } & \multicolumn{2}{c}{ Incontinence } & \multicolumn{2}{c}{ Voiding symptoms } & NO symptoms \\
& $\mathbf{0 - 1}$ & $\mathbf{> 2}$ & stress & urgency & $\mathbf{1}$ & $\mathbf{2}$ & \\
& 37 & 114 & 35 & 34 & 98 & 53 & 28 \\
\hline
\end{tabular}

Table 4 Characteristics of control population: mean values of each item are presented

$\begin{array}{lll}\text { Total number } \mathbf{n}=\mathbf{1 5 0} & \text { Age years } \mathbf{P}<\mathbf{0 . 0 5} & \text { BMI }(\mathrm{Kg} / \mathbf{m} 2) \mathbf{P}<\mathbf{0 . 0 1} \\ & 36 & 27\end{array}$

\section{Discussion}

Diabetic cystopathy is common and have a large spectrum of clinical presentations. It can be unnoted, but it is normally associated with a significant impact in quality of life. The two most frequent causes of incontinence are urgency incontinence (excessive pressure development by bladder) and stress incontinence (reduced bladder outlet resistance). ${ }^{4-19}$ Diabetes has been identified as an important independent risk factor for urinary incontinence that leads to distress and poorer quality of life. ${ }^{4-21}$ In our study, concerning diabetic population, storage symptoms were significantly associated with age, feminine sex, body mass index and diabetes type 2; we also found a statistically relation between chronic complications, like nephropathy, and bladder dysfunction symptoms. Recent studies demonstrated an association between metabolic syndrome and bladder dysfunction and we also found a high prevalence of bladder symptoms in patients with chronic complications and metabolic syndrome. Comparing to the control population, we conclude that diabetes can contribute for bladder dysfunction. The storage and voiding symptoms were significantly higher in diabetic patients than in controls. In our study, we have some limitations because our diabetic and control populations have some differences, concerning age (diabetic population is older) and BMI (which is higher in diabetic population), that could per si cause vesical symptoms. On the other hand, we did not ask to female population the parity that could also contribute to bladder dysfunction. Nearly $25-50 \%$ of patients do not complain of bladder problems unless specifically asked. Understanding the symptoms experienced by the patient, it is an important initial step. For proper evaluation of this pathology, many causes of vesico-urethral dysfunction should be excluded, as well as conducting a properly clinical history, including a questionnaire of urinary symptoms, in order to characterize the disease status. $^{8-23}$ If there is any suspicion of bladder dysfunction presence during asymptomatic phase, appropriate preventive and therapeutic approaches could be taken; furthermore the characterization of the disease is crucial to the therapeutic strategy option, which includes behavioral measures, pharmacological and rarely surgery. ${ }^{4-8}$

\section{Acknowledgments}

Nurse team: Carla Encarnação, Margarita Puga, Sandra Silva, Sofia Silva, Susana Azevedo, Vânia Silva.

\section{Conflict of Interest}

The authors declare there is no conflict of interest.

\section{References}

1. Karoli R, Bhat S, Fatima J, et al. A study of bladder dysfunction in women with type 2 diabetes mellitus. Indian $J$ Endocrinol Metab. 2014;18(4):552-557.

2. Yuan Z, Tang Z, He C. et al. Diabetic cystopathy: A review. Journal of Diabetes. 2015;7(4):442-447.

3. Brown JS, Wing R, Barrett ConnorE, et al. Lifestyle intervention is associated with lower prevalence of urinary incontinence: the Diabetes Prevention Program. Diabetes care. 2006;2:385-390.

4. Changxiao H, Zhengyong Y, Shibing Y. et al. Clinical and urodynamic evaluation of women referred with diabetes mellitus. Int Urogynecol $J$ Pelvic Floor Dysfunct. 2014;25(7):979-983.

5. Daneshgari F, Liu G, Birder L, et al. Diabetic Bladder Dysfunction: Current Translational Knowledge. J Urol. 2009;182(Suppl 6):18-26.

6. Gammack JK. Lower urinary tract symptoms. Clinics in Geriatric Medicine. 2010;26(2):249-260.

7. Wein AJ. An International Urogynecological Association (IUGA)/ International Continence Society (ICS) joint report on the terminology for female pelvic floor dysfunction. Journal of Urology. 2010;185(5):1812-2011.

8. Golbidi S, Laher I. Bladder dysfunction in diabetes mellitus. Front Pharmacol. 2010;1:136.

9. Gomez CS, Kanagarajah P, Gousse AE. Bladder dysfunction in patients with diabetes. Curr Urol Rep. 2011;12(6): 419-426.

10. Jose B, Karampini E, Pucci M, et al. Diabetic cystopathy: Do we recognise this in our patients. Pract Diabetes Int?. 2011;28(3):129-131. 
11. Jose B, Karampini E, Pucci M, et al. De Diabetic cystopathy: An unusual manifestation of autonomic dysfunction. Diabet Med. 2010;27(2):95.

12. Arrellano Valdez F, Urrutia Osorio M, Arroyo C, et al. A comprehensive review of urologic complications in patients with diabetes. Springerplus. 2014;3(1):549.

13. Kebapci N, Yenilmez A, Efe B, et al. Demirustu, Bladder dysfunction in type 2 diabetic patients. Neurourol Urodyn. 2007;26(6):814-819.

14. Lee WC, Wu CC, Wu HP, et al. Lower urinary tract symptoms and uroflowmetry in women with type 2 diabetes mellitus with and without bladder dysfunction. Urology. 2007;69(4):685-690.

15. Lin TL, Chen GD, Chen YC, et al. Aging and recurrent urinary tract infections are associated with bladder dysfunction in type 2 diabetes. Taiwan J Obstet Gynecol. 2012;51(3):381-386.

16. Liu G, Daneshgari F. Diabetic bladder dysfunction. Chinese Medical Journal. 2014;127(7): 1357-1364.
17. Miyazato M, Yoshimura N, Chancellor MB. The other bladder syndrome: underactive bladder. Rev Urol. 2013;15(1):11-22.

18. Nanigian DK, Keegan KA, Stone AR. Diabetic cystopathy. Curr Bladder Dysfunct Rep. 2007;2(4):197-202.

19. Rapidi CA, Karandreas N, Katsifotis C, et al. A combined urodynamic and electrophysiological study of diabetic cystopathy, Neurourol. Urodyn. 2006;25(1):32-38.

20. Tong Y, Jia Q, Sun Y, et al. Acupuncture in the treatment of diabetic bladder dysfunction. J Altern Complement Med. 2009;15:905-909.

21. Gaur C, Mathur A, Agarwal A, et al. Diabetic autonomic neuropathy causing gall bladder dysfunction. $J$ Assoc Physicians India. 2000;48:603-605.

22. Xiao N, Wang Z, Huang Y, et al. Roles of polyuria and hyperglycemia in bladder dysfunction in diabetes. J Urol. 2013;189(3):1130-1136. 